Full Paper

\title{
Untersuchungen zum Vorkommen von Phytoseiidae (Acari: Mesostigmata) und Roten Spinne, Panonychus ulmi im Südtiroler Apfelanbau
}

\author{
Survey of phytoseiid mites (Acari: Mesostigmata) and the European red mite Panonychus ulmi Koch in \\ apple orchards of South Tyrol, Northern Italy
}

Monitoraggio delle popolazioni di Panonychus ulmi e indagini faunistiche pluriennali sui fitoseidi nei meleti dell'Alto Adige

Martin Parth ${ }^{1}$, Stefanie Fischnaller ${ }^{1}$, Manuel Messner ${ }^{1}$, Manfred Wolf ${ }^{1}$

${ }^{1}$ Versuchszentrum Laimburg

\section{ABSTRACT}

In order to get detailed information about the population dynamics and densities of the tetranychid pest Panonychus ulmi (Koch 1836) and phytoseiid mites in apple orchards in South Tyrol (Northern Italy), investigations were carried out during four consecutive vegetation periods in 50 sites. Furthermore, a faunistic analysis of mite species, present during summer and wintertime, was realized.

A total of seven phytoseiid species were found, whereas Amblyseius andersoni (Chant 1957) was an eudominant and the most frequent species on leaves and in branch-stub winter shelters. A. andersoni showed ubiquitous occurence and represented $80 \%$ of total determined specimens. A permanently presence of $A$. andersoni was observed in $85 \%$ of the orchards. Typhlodromus pyri (Scheuten 1857) and Euseius finlandicus (Oudemans 1915) were permanently present at $18.5 \%$ and $7.4 \%$ of the locations and their quantity constitute $12.8 \%$ and $5.8 \%$ of total phytoseiid count, respectively. Neoseiulus californicus (McGregor 1954) was found for the first time on apple trees in South Tyrol. Kampimodromus aberrans (Oudemans 1930), Paraseiulus talbii (Athias-Henriot 1960) and Typhlodromus bakeri (Garman 1948) occured sporadically and represented altogether less than $2 \%$ of total classified phytoseiid records.

\section{KEYWORDS}

Apple, faunistic survey, Panonychus ulmi, Phytoseiidae, predatory mites, South Tyrol

\section{CITE ARTICLE AS}

Parth Martin, Fischnaller Stefanie, Messner Manuel et.al. (2020). Survey of phytoseiid mites (Acari: Mesostigmata) and the European red mite Panonychus ulmi Koch in apple orchards of South Tyrol, Northern Italy.

Laimburg Journal 02/2020

DOI: $10.23796 /$ LJ/2020.008

\section{CORRESPONDING AUTHOR}

Martin Parth

Laimburg 6, Pfatten, I-39040 Auer

(BZ), Italien

martin.parth@laimburg.it +390471969744 


\section{EINLEITUNG}

Aufgrund des vermehrten großflächigen Einsatzes von raubmilbenschädlichen Präparaten bei der Bekämpfung der Hauptvektoren der Apfeltriebsucht in Südtirol (ab 2013), wird befürchtet, dass das Auftreten von Schadmilbenbefall zunehmen könnte [1]. Damit rücken das Interesse für die Raubmilben und der Bedarf an gezielten Untersuchungen wieder in den Vordergrund.

Durch Eingriffe im Zuge der Produktion, insbesondere durch den Einsatz der genannten Pflanzenschutzmaßnahmen, aber auch durch diverse Umweltfaktoren kann die Populationsdynamik von Raub- und Schadmilben gestört und damit das natürliche Gleichgewicht dieser Räuber-Beute-Dynamik negativ beeinflusst werden [2] [3] [4].

Ziel der mehrjährig angelegten Untersuchungen war die Überwachung der Populationsdichten von P. Ulmi und Raubmilben in den Apfelanbaugebieten des Etschtals und Vinschgaus. Aufgrund der lückenhaften Kenntnis bezüglich des aktuell auf Apfel vorkommenden Artenspektrums der Raubmilben, wurde weiters eine mehrjährige faunistische Untersuchung der Phytoseiidae durchgeführt [5] [6].

\section{MATERIAL UND METHODEN}

In die Untersuchungen (2014-2017) wurden insgesamt 50 Apfelanlagen einbezogen. Bei den Untersuchungsflächen handelte es sich überwiegend um integriert bewirtschaftete Ertragsanlagen im Burggrafenamt und Vinschgau (Abb. 1, Tab. 1). Zur Erhebung der Populationsdichten wurden Blatt-Beprobungen im Juni, Juli sowie im August vorgenommen. Aus Gründen der Vergleichbarkeit beschränkt sich die vorliegende Auswertung auf Daten aus Anlagen bzw. Sorten, welche in allen untersuchten Südtiroler Obstbaulagen vorkamen. Die faunistische Analyse wurde daher in einer Unterauswahl von 27 Anlagen, der Sorten Gala und Golden Delicious über die Jahre 2015 bis 2017 durchgeführt (Abb. 1, Tab. 1). Blattproben vom Juli sowie Astproben, welche im Dezember gesammelt wurden, bildeten die Grundlage der Faunistik.

Jede Einzelprobe bestand aus zufällig entnommenen 100 Blättern. Es wurde stets nur ein Blatt pro Baum, aus der Mitte eines Fruchttriebs, im Höhenbereich von 1-1,8 m entnommen.

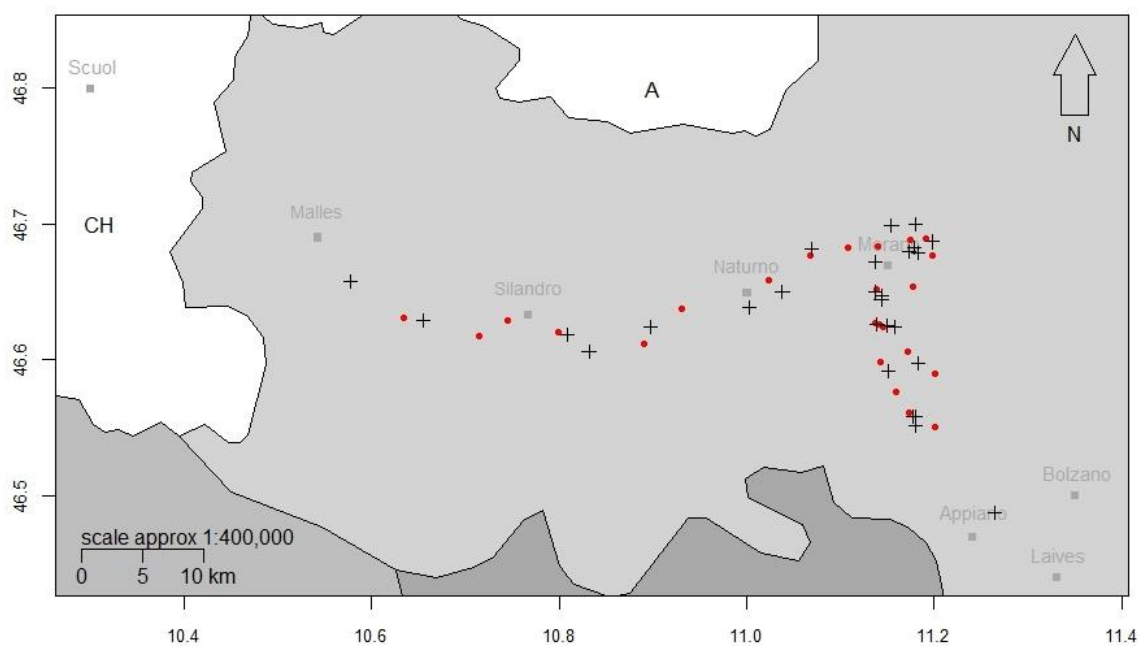

Abb. 1: Lage der untersuchten Apfelanlagen im Etschtal und Vinschgau: Erhebungsstandorte, an welchen die Populationsdichten von Raubmilben und $P$. ulmi bestimmt wurden sind jeweils durch einen roten Punkt oder ein schwarzes Kreuz gekennzeichnet. Apfelanlagen, welche faunistisch untersucht wurden sind durch ein Kreuz markiert. Geographisches Koordinatensystem WGS 84 mit Längengraden (Ost) auf der Abszissenachse und Breitengraden auf der Ordinatenachse (Nord) [Dezimalgrade]. // Map of Vinschgau Valley and Adige Valley with geographic positions of investigated apple orchards: population density study sites are marked either by crosses or points. Crosses are marking the faunistic study sites. WGS 84 Coordinate System with longitude (E) on the $X$-axis and latitude ( $N$ ) on the $Y$-axis [decimal degrees].

Die Beprobung am „alten Holz“ erfolgte jeweils im Dezember während der Vegetationsruhe im Winter. Die im Kronenbereich des Apfels überwinternden Raubmilbenweibchen können so beprobt werden. Die Aststücke wurden endständig von bestehenden Aststummeln (Ergebnis zurückliegender Schnitttätigkeit) abgeschnitten. Dabei wurden jeweils 80 Aststücke pro Anlage und Termin gesammelt. Es wurde jeweils nur ein Aststück mit einem Durchmesser 0,5-1,5 cm pro Baum entnommen. Die Entnahme erfolgte stets in zwei ausgewählten Reihen (einmalige zufällige Auswahl) in einem festgelegten Abstand: Abhängig von der Reihenlänge wurde entweder jeder oder jeder zweite Baum entlang einer Reihe beprobt.

Die Astproben wurden unmittelbar nach Probennahme in dichtschließende Schraubgefäße überführt und für drei Tage bei einer Temperatur $\geq 25{ }^{\circ} \mathrm{C}$ inkubiert, um die Winterruhe der Raubmilben zu brechen und das Auswandern der Milben zu forcieren.

Die Aufarbeitung von Blatt- und Astproben erfolgte mittels einer adaptierten Waschmethode nach Boller, 1984 [7]. Das Pflanzenmaterial wurde über Nacht in einer Tensidlösung $\left[0,7 \% \mathrm{v} / \mathrm{v}\right.$ Irol ${ }^{\circledR}$ (Siapa, Italy)] getränkt und schließlich mithilfe eines Wasserstrahls gespült. Der Überstand und die Waschlösung wurden schließlich mittels ei- nes groben Siebes und einer nachgeschalteten Nylonmembran $(0,1 \mathrm{~mm})$ filtriert. Der Filtrationsrückstand wurde in eine $70 \%$ EtOH-Lösung überführt. Anschließend wurden die im Filtrationsrückstand befindlichen Raubmilben sowie $P$. ulmi-Individuen mithilfe eines Stereomikroskopes ausgezählt.

Zur Artbestimmung wurden temporäre Präparate angefertigt: Raubmilbenexemplare wurden über zwei Stunden bei $70{ }^{\circ} \mathrm{C}$ in $96 \%$ v/v Milchsäure gebleicht und anschließend auf Objektträgern in 15\% v/v Milchsäure eingebettet. Die taxonomische Bestimmung wurde mittels Phasenkontrastmikroskops bei 600-facher Vergrößerung vorgenommen. Die Klassifikation erfolgte anhand der Bestimmungsschlüssel von Hernandes et al., 2012 [8], Miediema, 1987 [9] und Tixier et al., 2013 [10]. Die hier verwendete Nomenklatur orientiert sich an der Phytoseiidae Database von Demite et al., 2019 [11].

Die Raubmilben und das daraus ermittelte Artenspektrum wurde getrennt nach Jahren und Probennahme hinsichtlich Dominanz und Frequenz untersucht.

Die Frequenz $(F)$ ist ein Maß für die Verbreitung einer Art im Untersuchungsgebiet und ergibt sich wie folgt:

$\mathrm{F}=($ Anzahl der Fundorte einer Art $i \times 100) /$ Gesamtzahl an Untersuchungsflächen; [\%]. 
Tab. 1: Probenstandorte der faunistischen Untersuchung: Standortbezeichnung (a-ß), Höhenlage, Koordinaten, Bewirtschaftungsform, Sorte und Beprobungszeitraum. Integrated*: Integrierte Produktion ohne Insektizideinsatz, Integrated: Integrierte Produktion, Organic: Biologische Produktion. // Location information of the faunistic study sites. Integrated*: integrated production without insecticides.

\begin{tabular}{|c|c|c|c|c|c|}
\hline \multicolumn{6}{|c|}{$\begin{array}{l}\text { Übersicht zur geographischen Lage und Produktionsweise der untersuchten Apfelanlagen } \\
\text { Overview of geographical position and production method of sampling sites }\end{array}$} \\
\hline $\begin{array}{c}\text { Anlagenkürzel } \\
\text { Identification letter of } \\
\text { sampling sites }\end{array}$ & $\begin{array}{c}\text { Höhe } \\
\text { Altitude } \\
\text { (m a.s.l.; +/- } 25 \mathrm{~m})\end{array}$ & $\begin{array}{c}\text { Koordinaten } \\
\text { Geographic coordinates } \\
\text { [WGS 84: latitude (N); longi- } \\
\text { tude (E)] }\end{array}$ & $\begin{array}{c}\text { Anbauweise } \\
\text { Orchard management }\end{array}$ & $\begin{array}{l}\text { Sorte } \\
\text { Variety }\end{array}$ & $\begin{array}{l}\text { Beprobungs- } \\
\text { zeitraum } \\
\text { Survey years }\end{array}$ \\
\hline \multicolumn{6}{|c|}{ Situated in Vinschgau Valley: } \\
\hline a & 900 & $46.657659^{\circ} ; 10.577335^{\circ}$ & Integrated* & Golden Delicious & 2015-2017 \\
\hline b & 900 & $46.629361^{\circ} ; 10.654882^{\circ}$ & Organic & Golden Delicious & 2015-2017 \\
\hline c & 700 & $46.618487^{\circ} ; 10.808258^{\circ}$ & Integrated & Golden Delicious & 2015-2017 \\
\hline$d$ & 650 & $46.606275^{\circ} ; 10.831569^{\circ}$ & Integrated & Golden Delicious & 2015-2017 \\
\hline e & 650 & $46.624276^{\circ} ; 10.897132^{\circ}$ & Integrated & Golden Delicious & $2015-2017$ \\
\hline$f$ & 600 & $46.638432^{\circ} ; 11.002301^{\circ}$ & Int. /Org. since 2017 & Gala & 2015-2017 \\
\hline g & 550 & $46.650257^{\circ} ; 11.037999^{\circ}$ & Integrated & Golden Delicious & 2015-2017 \\
\hline $\mathrm{h}$ & 650 & $46.681952^{\circ} ; 11.069166^{\circ}$ & Integrated & Golden Delicious & 2015-2017 \\
\hline \multicolumn{6}{|c|}{ Situated in Adige Valley: } \\
\hline $\mathrm{i}$ & 600 & $46.686921^{\circ} ; 11.198136^{\circ}$ & Integrated & Golden Delicious & 2015-2017 \\
\hline j & 400 & $46.678519^{\circ} ; 11.182662^{\circ}$ & Integrated & Golden Delicious & $2015-2017$ \\
\hline k & 370 & $46.682323^{\circ} ; 11.178261^{\circ}$ & Integrated & Golden Delicious & $2015-2017$ \\
\hline 1 & 370 & $46.679790^{\circ} ; 11.173554^{\circ}$ & Integrated & Golden Delicious & $2015-2017$ \\
\hline $\mathrm{m}$ & 500 & $46.699772^{\circ} ; 11.179915^{\circ}$ & Integrated & Golden Delicious & 2015-2017 \\
\hline $\mathrm{n}$ & 700 & $46.699164^{\circ} ; 11.154460^{\circ}$ & Integrated & Golden Delicious & 2015-2017 \\
\hline o & 350 & $46.672175^{\circ} ; 11.137174^{\circ}$ & Integrated & Gala & 2015-2017 \\
\hline $\mathrm{p}$ & 300 & $46.644695^{\circ} ; 11.143432^{\circ}$ & Integrated & Golden Delicious & $2015-2017$ \\
\hline$q$ & 300 & $46.647199^{\circ} ; 11.143679^{\circ}$ & Integrated & Golden Delicious & 2015-2017 \\
\hline r & 450 & $46.650054^{\circ} ; 11.136713^{\circ}$ & Integrated & Golden Delicious & 2015-2017 \\
\hline s & 400 & $46.626491^{\circ} ; 11.138599^{\circ}$ & Integrated & Golden Delicious & 2015-2017 \\
\hline $\mathrm{t}$ & 300 & $46.625071^{\circ} ; 11.149663^{\circ}$ & Organic & Gala & $2015-2017$ \\
\hline $\mathrm{u}$ & 300 & $46.624566^{\circ} ; 11.158074^{\circ}$ & Integrated & Golden Delicious & 2015-2017 \\
\hline$v$ & 250 & $46.597541^{\circ} ; 11.182721^{\circ}$ & Integrated & Gala & 2015-2017 \\
\hline w & 700 & $46.591898^{\circ} ; 11.151089^{\circ}$ & Integrated & Golden Delicious & $2015-2017$ \\
\hline$x$ & 580 & $46.558197^{\circ} ; 11.177180^{\circ}$ & Organic & Gala & 2016-2017 \\
\hline y & 590 & $46.557856^{\circ} ; 11.179513^{\circ}$ & Organic & Gala & 2016-2017 \\
\hline z & 600 & $46.551228^{\circ} ; 11.179777^{\circ}$ & Integrated & Golden Delicious & $2015-2017$ \\
\hline$\beta$ & 250 & $46.487279^{\circ} ; 11.264795^{\circ}$ & Integrated & Gala & $2015-2017$ \\
\hline
\end{tabular}


Die Dominanz (D) beschreibt den relativen Anteil einer Art an der Gesamtzahl an Individuen aller Arten bzw. eines Taxons (hier Phytoseiidae) in einer Biozönose [12] [13] [14]:

$\mathrm{D}=$ (Individuenzahl einer Art $i \times 100) /$ Individuenzahl der Gesamtheit aller Arten; [\%].

Zur Bewertung der Dominanzstruktur erfolgte eine Einordnung der Arten in Dominanzklassen gemäß Engelmann, 1978 [12]:

\begin{tabular}{|c|c|c|}
\hline$D=$ & $100 \%-32 \%$ & Eudominant \\
\hline & $31,9 \%-10 \%$ & Dominant \\
\hline & $10 \%-3,2 \%$ & Subdominant \\
\hline & $3,1 \%-1 \%$ & Rezedent \\
\hline & $0,9 \%-0,32 \%$ & Subrezedent \\
\hline & $<0,32 \%$ & Sporadisch \\
\hline
\end{tabular}

Bei der Abundanz handelt es sich um die durchschnittliche Zahl der Individuen einer Art bzw. eines Taxons, bezogen auf eine Flächen- oder Raumeinheit (= Individuendichte) [15]. Die Abundanz wird im Folgenden als Individuenzahl pro Blatt bzw. Individuenzahl pro Schnittstelle angegeben.

\section{ERGEBNISSE}

\section{FAUNISTIK}

Im Zuge der faunistischen Untersuchung in 27 Anlagen wurden 5581 adulte Raubmilben-Individuen auf Artniveau klassifiziert. 3057 dieser Individuen stammten aus Blattproben, während 2524 aus Astproben der Winterperioden gesammelt wurden (Tab. 2).

Es konnten sieben Arten der Familie der Phytoseiidae nachgewiesen werden. Bei den festgestellten Arten handelt es sich um Vertreter folgender sechs Genera: Amblyseius (Berlese 1904), Euseius (Wainstein 1962), Neoseiulus (Hughes 1948), Paraseiulus (Muma 1961), Kampimodromus (Nesbitt 1951) und Typhlodromus (Scheuten 1857).

Vier Arten wurden sowohl auf den Blattproben als auch in den Astproben nachgewiesen: Amblyseius andersoni (Chant), Euseius finlandicus (Oudemans), Paraseiulus talbii (Athias-Henriot) und Typhlodromus pyri (Scheuten). Exemplare von Neoseiulus californicus (McGregor) und Kampimodromus aberrans (Oudemans) wurden hingegen ausschießlich am Blatt gefunden, während sich Typhlodromus bakeri (Garman) nur in einer Astprobe fand.

Im Juli 2017 wurden 16 weibliche Exemplare von $N$. californicus in zwei Apfelanlagen im Raum Marling gefunden. Es ist dies der erstmalig beschriebene Nachweis dieser Art am Apfel in Südtirol. Die beiden Fundorte liegen in einer ungefähren Luftlinienentfernung von $650 \mathrm{~m}$ auseinander (Anlagen q, r; Tab. 1). Im Winter konnten hingegen keine Exemplare von $N$. californicus gefunden werden.

K. aberrans wurde mit sieben Individuen ausschließlich in Blattproben festgestellt. Der Medianwert der Frequenz lag über den Erhebungszeitraum bei $9 \%$.

T. bakeri wurde einmalig mit zwei Exemplaren an Ästen einer biologisch bewirtschafteten Anlage gefunden.

P. talbii wurde 2015 lediglich in Astproben nachgewiesen; 2016 und 2017 wurde die Art auch in den Blattproben gefunden (Abb. 2). In Summe wurden 47 Individuen gesammelt. Die Frequenz von $P$. talbii lag gemittelt bei $4 \%$ anhand von Blattproben im Sommer beziehungsweise bei $18 \%$ im Winter.

A. andersoni erwies sich mit einer Dominanzrate von $75 \%$ an Blättern und $86 \%$ an Astproben als eudominante und eukonstante Art im Untersuchungsgebiet. Sie trat an jedem der Untersuchungsorte wenigstens einmalig auf (Tab. 2, Tab. 3). An drei
Standorten war sie die einzige nachweisbare Art. In den Anlagen des Burggrafenamts wurde $A$. andersoni in sämtlichen Raubmilben-enthaltenden Proben nachgewiesen (Anlagen $\mathrm{f}-\mathrm{z}$; Tab. 3). Insgesamt wurden 4469 Exemplare als $A$. andersoni klassifiziert, dies entspricht einem Anteil von 80,1\% an der Individuen-Gesamtzahl.

T. pyri erwies sich als zweithäufigste Art sowohl an Blättern als auch an Ästen (Abb. 2). Ihr Dominanzindexwert anhand von Blattproben beläuft sich auf $16 \%$ und untersteicht damit die dominante Stellung dieser Art [12]. An Ästen hingegen zeigte T. pyri ein subdominantes Vorkommen ( $D<10 \%,[12])$. Gemessen an Blattproben beläuft sich die mittlere Frequenz von $T$. pyri auf $37 \%$, anhand von Astproben auf 24\%. Die Abundanz von T. pyri erschien jedoch geografisch inhomogen: Die Art nahm vor allem in höheren bis mittleren Anlagen des Vinschgaus eine dominante Rolle ein, während im restlichen Gebiet fast ausschließlich A. andersoni dominierte.

E. finlandicus trat sowohl an Blättern als auch im Winter in subdominanter Weise auf. Über die Winterbeprobung konnte eine Frequenz von $29 \%$ ermittelt werden, welche sich damit höher ausgeprägt zeigte als die Sommer-Frequenz an Blättern (19\%).

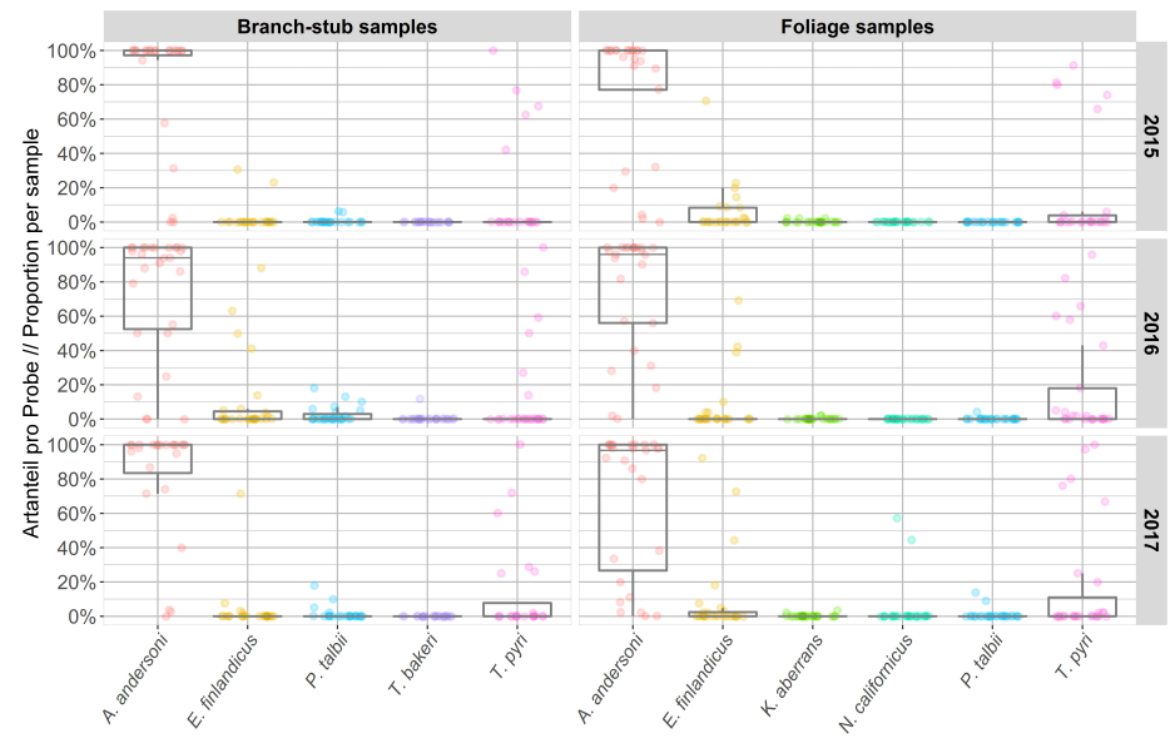

Abb. 2: Raubmilben-Artanteil pro Probe. Branch-stub samples: Astproben; Foliage samples: Blattproben. // Species proportion per sample according to sampling type and year. 
Tab. 2: Individuenzahl und Dominanz der nachgewiesenen Raubmilbenarten aufgeschlüsselt nach Probenart und Jahr. // Specimen number and dominance of detected phytoseiid species according to sampling method and year.

\begin{tabular}{|c|c|c|c|c|c|c|}
\hline & \multirow{2}{*}{\multicolumn{3}{|c|}{$\begin{array}{l}\text { Anzahl klassifizierter Raubmilben } \\
\text { Number of classified specimens }\end{array}$}} & \multicolumn{3}{|c|}{ Dominanz } \\
\hline & & & & \multicolumn{3}{|c|}{ Species dominance } \\
\hline & 2015 & 2016 & 2017 & 2015 & 2016 & 2017 \\
\hline \multicolumn{7}{|c|}{ Blattproben // Foliage samples } \\
\hline Amblyseius andersoni & 680 & 841 & 785 & $74,2 \%$ & $77,7 \%$ & $74,2 \%$ \\
\hline Euseius finlandicus & 60 & 77 & 93 & $6,6 \%$ & $7,1 \%$ & $8,8 \%$ \\
\hline Kampimodromus aberrans & 3 & 2 & 2 & $0,3 \%$ & $0,2 \%$ & $0,2 \%$ \\
\hline Neoseiulus californicus & 0 & 0 & 16 & $0,0 \%$ & $0,0 \%$ & $1,5 \%$ \\
\hline Paraseiulus talbii & 0 & 2 & 11 & $0,0 \%$ & $0,2 \%$ & $1,0 \%$ \\
\hline Typhlodromus bakeri & 0 & 0 & 0 & $0,0 \%$ & $0,0 \%$ & $0,0 \%$ \\
\hline Typhlodromus pyri & 173 & 161 & 151 & $18,9 \%$ & $14,9 \%$ & $14,3 \%$ \\
\hline \multicolumn{7}{|c|}{ Astproben // Branch-stub samples } \\
\hline Amblyseius andersoni & 723 & 721 & 719 & $87,3 \%$ & $82,2 \%$ & $87,8 \%$ \\
\hline Euseius finlandicus & 20 & 48 & 25 & $2,4 \%$ & $5,5 \%$ & $3,1 \%$ \\
\hline Kampimodromus aberrans & 0 & 0 & 0 & $0,0 \%$ & $0,0 \%$ & $0,0 \%$ \\
\hline Neoseiulus californicus & 0 & 0 & 0 & $0,0 \%$ & $0,0 \%$ & $0,0 \%$ \\
\hline Paraseiulus talbii & 4 & 18 & 12 & $0,5 \%$ & $2,1 \%$ & $1,5 \%$ \\
\hline Typhlodromus bakeri & 0 & 2 & 0 & $0,0 \%$ & $0,2 \%$ & $0,0 \%$ \\
\hline Typhlodromus pyri & 81 & 88 & 63 & $9,8 \%$ & $10,0 \%$ & $7,7 \%$ \\
\hline
\end{tabular}


Tab. 3: Nachgewiesene Raubmilbenarten an den Untersuchungsflächen: Artenvorkommen an Blättern sowie an Astproben aufgeschlüsselt nach Anlage (Anlagenbezeichnung gemäß Tab. 1) und Jahr. Aa: Amblyseius andersoni, Ef: Euseius finlandicus, Ka: Kampimodromus aberrans, Nc: Neoseiulus californicus, Pt: Paraseiulus talbii, Tb: Typhlodromus bakeri, Tp: Typhlodromus pyri, n.a.: keine Daten, /: keine Raubmilben gefunden. // Register of occuring phytoseiid species according to sampling method, sampling site and year. Aa: Amblyseius andersoni, Ef: Euseius finlandicus, Ka: Kampimodromus aberrans, Nc: Neoseiulus californicus, Pt: Paraseiulus talbii, Tb: Typhlodromus bakeri, Tp: Typhlodromus pyri, n.a.: not available, /: no specimens.

\begin{tabular}{|c|c|c|c|c|c|c|}
\hline & \multicolumn{6}{|c|}{$\begin{array}{l}\text { Vorkommende Raubmilbenarten } \\
\text { Occuring pytoseiid species }\end{array}$} \\
\hline & \multicolumn{3}{|c|}{$\begin{array}{l}\text { Blattproben } \\
\text { Foliage samples }\end{array}$} & \multicolumn{3}{|c|}{$\begin{array}{c}\text { Astproben } \\
\text { Branch-stub samples }\end{array}$} \\
\hline $\begin{array}{l}\text { Anlage } \\
\text { Site }\end{array}$ & 2015 & 2016 & 2017 & 2015 & 2016 & 2017 \\
\hline a & $\mathrm{Aa}, \mathrm{Tp}$ & $\mathrm{Aa}, \mathrm{Tp}$ & $\mathrm{Aa}, \mathrm{Tp}$ & $\mathrm{Aa}$ & $\mathrm{Aa}, \mathrm{Tp}$ & $\mathrm{Aa}, \mathrm{Tp}$ \\
\hline$b$ & $\mathrm{Aa}, \mathrm{Tp}$ & $\mathrm{Aa}, \mathrm{Tp}$ & $\mathrm{Tp}$ & $\mathrm{Tp}$ & $T p$ & $\mathrm{Tp}$ \\
\hline c & $E f, T p$ & $\mathrm{Aa}, \mathrm{Tp}$ & Ef, Tp & $\mathrm{Aa}, \mathrm{Pt}, \mathrm{Tp}$ & $\mathrm{Aa}, \mathrm{Tp}$ & $\mathrm{Aa}, \mathrm{Tp}$ \\
\hline$d$ & $\mathrm{Aa}$ & $\mathrm{Aa}, \mathrm{Tp}$ & $\mathrm{Aa}, \mathrm{Pt}$ & $\mathrm{Aa}$ & $\mathrm{Aa}, \mathrm{Pt}$ & $\mathrm{Aa}$ \\
\hline e & $\mathrm{Aa}, \mathrm{Ef}, \mathrm{Ka}, \mathrm{Tp}$ & $\mathrm{Ef}, \mathrm{Tp}$ & $\mathrm{Aa}, \mathrm{Ef}, \mathrm{Tp}$ & $\mathrm{Aa}, \mathrm{Ef}, \mathrm{Tp}$ & $T p, E f$ & $\mathrm{Aa}, \mathrm{Ef}, \mathrm{Tp}$ \\
\hline$f$ & $\mathrm{Aa}$ & $\mathrm{Aa}$ & $\mathrm{Aa}$ & $\mathrm{Aa}$ & $\mathrm{Aa}$ & / \\
\hline g & $\mathrm{Aa}$ & $\mathrm{Aa}, \mathrm{Tp}$ & $\mathrm{Aa}$ & $\mathrm{Aa}$ & $\mathrm{Aa}$ & $\mathrm{Aa}$ \\
\hline $\mathrm{h}$ & $\mathrm{Aa}$ & $\mathrm{Aa}, \mathrm{Tp}$ & $\mathrm{Aa}, \mathrm{Tp}$ & $\mathrm{Aa}$ & $\mathrm{Aa}, \mathrm{Ef}, \mathrm{Pt}$ & $\mathrm{Aa}$ \\
\hline $\mathrm{i}$ & $\mathrm{Aa}$ & $\mathrm{Aa}$ & $\mathrm{Aa}, \mathrm{Ka}$ & $\mathrm{Aa}$ & $\mathrm{Aa}$ & $\mathrm{Aa}$ \\
\hline j & $\mathrm{Aa}$ & $A a, E f, T p$ & $\mathrm{Aa}$ & $\mathrm{Aa}$ & $\mathrm{Aa}$ & $\mathrm{Aa}$ \\
\hline k & $\mathrm{Aa}, \mathrm{Ef}, \mathrm{Tp}$ & $\mathrm{Aa}$ & $\mathrm{Aa}, \mathrm{Tp}$ & $\mathrm{Aa}$ & $\mathrm{Aa}$ & $\mathrm{Aa}$ \\
\hline I & $\mathrm{Aa}$ & $\mathrm{Aa}$ & $\mathrm{Aa}$ & $\mathrm{Aa}$ & $\mathrm{Aa}$ & $\mathrm{Aa}$ \\
\hline $\mathrm{m}$ & $\mathrm{Aa}$ & $\mathrm{Aa}$ & $A a, E f$ & $\mathrm{Aa}$ & $\mathrm{Aa}, \mathrm{Ef}$ & $\mathrm{Aa}, \mathrm{Ef}, \mathrm{Pt}$ \\
\hline $\mathrm{n}$ & $\mathrm{Aa}, \mathrm{Ef}$ & $\mathrm{Aa}, \mathrm{Ef}$ & $\mathrm{Aa}, \mathrm{Ef}$ & n.a. & $\mathrm{Aa}, \mathrm{Ef}$ & $\mathrm{Aa}$ \\
\hline o & $\mathrm{Aa}$ & $\mathrm{Aa}$ & $\mathrm{Aa}$ & $\mathrm{Aa}, \mathrm{Pt}$ & $\mathrm{Aa}$ & $\mathrm{Aa}, \mathrm{Pt}$ \\
\hline $\mathrm{p}$ & $\mathrm{Aa}$ & $\mathrm{Aa}$ & $\mathrm{Aa}, \mathrm{Tp}$ & $\mathrm{Aa}$ & $\mathrm{Aa}$ & $\mathrm{Aa}$ \\
\hline$q$ & $\mathrm{Aa}, \mathrm{Ef}, \mathrm{Ka}$ & $A a, E f$ & $\mathrm{Aa}, \mathrm{Ef}, \mathrm{Nc}$ & $\mathrm{Aa}$ & $\mathrm{Aa}, \mathrm{Ef}, \mathrm{Pt}$ & n.a. \\
\hline$r$ & $\mathrm{Aa}, \mathrm{Ef}$ & $A a, E f, T p$ & $\mathrm{Aa}, \mathrm{Ef}, \mathrm{Nc}$ & / & $\mathrm{Aa}, \mathrm{Ef}$ & n.a. \\
\hline s & $A a, E f$ & $A a, E f$ & $\mathrm{Aa}$ & $\mathrm{Aa}$ & $A a, E f$ & $\mathrm{Aa}$ \\
\hline $\mathrm{t}$ & $A a, E f, T p$ & $\mathrm{Aa}, \mathrm{Ka}, \mathrm{Pt}, \mathrm{Tp}$ & $\mathrm{Aa}, \mathrm{Ef}, \mathrm{Ka}, \mathrm{Tp}$ & $\mathrm{Aa}, \mathrm{Tp}$ & $\mathrm{Aa}, \mathrm{Pt}, \mathrm{Tp}$ & $\mathrm{Aa}, \mathrm{Tp}$ \\
\hline u & $\mathrm{Aa}$ & $\mathrm{Aa}$ & $\mathrm{Aa}, \mathrm{Tp}$ & $\mathrm{Aa}$ & $\mathrm{Aa}, \mathrm{Ef}, \mathrm{Pt}$ & $\mathrm{Aa}$ \\
\hline v & $\mathrm{Aa}$ & $\mathrm{Aa}$ & $\mathrm{Aa}$ & $\mathrm{Aa}$ & $\mathrm{Aa}$ & $\mathrm{Aa}, \mathrm{Pt}$ \\
\hline w & $\mathrm{Aa}, \mathrm{Tp}$ & $\mathrm{Aa}$ & $\mathrm{Aa}, \mathrm{Pt}$ & $\mathrm{Aa}$ & $\mathrm{Aa}, \mathrm{Pt}$ & $\mathrm{Aa}$ \\
\hline$x$ & n.a. & n.a. & $\mathrm{Aa}$ & n.a. & $\mathrm{Aa}, \mathrm{Tb}$ & $\mathrm{Aa}$ \\
\hline y & n.a. & n.a. & $\mathrm{Aa}$ & n.a. & $\mathrm{Aa}$ & $\mathrm{Aa}$ \\
\hline$z$ & $\mathrm{Aa}$ & $\mathrm{Aa}, \mathrm{Tp}$ & $\mathrm{Aa}$ & $\mathrm{Aa}$ & $\mathrm{Aa}, \mathrm{Pt}$ & $\mathrm{Aa}, \mathrm{Ef}, \mathrm{Tp}$ \\
\hline B & $\mathrm{Aa}, \mathrm{Ef}, \mathrm{Ka}, \mathrm{Tp}$ & $\mathrm{Aa}, \mathrm{Ka}, \mathrm{Tp}$ & Ef & $\mathrm{Ef}, \mathrm{Tp}$ & $\mathrm{Ef}, \mathrm{Pt}, \mathrm{Tp}$ & $\mathrm{Aa}, \mathrm{Ef}, \mathrm{Pt}, \mathrm{Tp}$ \\
\hline
\end{tabular}




\section{RAUBMILBENABUNDANZ}

In der überwiegenden Zahl der 50 Anlagen konnten stetige Raubmilbenpopulationen nachgewiesen werden. Die geringsten Dichten wurden im Monat Juni verzeichnet (vierjähriges Mittel = 0,63 Individuen pro Blatt) während der Populationshöhepunkt im Juli lag (vierjähriges Mittel = 0,77 Individuen pro Blatt).

Im Sommer 2014 wurde im Vergleich zu den drei Folgejahren ein äußerst geringer Raubmilben-Blattbesatz festgestellt: Der Median des Drei-Monatsdurchschnitts (Juni, Juli und August) lag bei 0,17 Individuen pro Blatt (Max $=0,7$, Abb. 3 ) und in $70 \%$ der Anlagen wurde an jedem Erhebungszeitpunkt eine Raubmilbendichte $<0,5$ Individuen pro Blatt verzeichnet. Im Juni 2014 wurde eine mittlere Dichte von lediglich 0,08 Individuen pro Blatt festgestellt, dieser Wert stieg im August auf 0,27 Individuen pro Blatt. Im Folgejahr 2015 konnte eine Besatzsituation mit $<0,5$ Individuen pro Blatt lediglich in 30\% der Anlagen beobachtet werden, wobei vor allem im Vinschgau geringere Dichten vorherrschten. Der Median des durchschnittlichen Raubmilbenbesatzes lag im Sommer 2015 bei 0,46 Individuen pro Blatt ( $M a x=$ 1,4). Im Jahr 2016 wurde die durchschnittlich höchste Blatt-bezogene Populationsdichte an Raubmilben festgestellt: Der Median des Drei-Monatsdurchschnitts lag bei 1,07 Individuen pro Blatt ( $\operatorname{Max}=4,73$ ).

Im Sommer 2017 wurde in der Mehrzahl der Anlagen ein durchschnittlicher Blattbesatz von $>0,5$ Individuen festgestellt (Median = 0,72 ; Max $=3,86$ ). Damit lag die generelle Populationsdichte der Raubmilben über jener der Jahre 2014 und 2015, erreichte jedoch nicht das Niveau von 2016 (Abb. 3).

\section{POPULATIONSDICHTE PA-} NONYCHUS ULMI

Das durchschnittlich geringste Aufkommen von P. ulmi wurde im Jahr 2014 verzeichnet: Der Median lag über die Sommermonate bei 0,003 Individuen pro Blatt ( $M a x=13,5, A b b$. 3) und in zwei von 50 Anlagen wurde eine durchschnittliche Abundanz $>2$ Individuen pro Blatt festgestellt.

Im Biennium 2015-2016 konnte eine durchschnittlich niedrige Populationsdichte homogener Ausprägung festgestellt werden (2015: Median =0,01, Max =1,11; 2016: Median $=0,01 ; \operatorname{Max}=1,19$ ), welche jedoch über jener von 2014 lag.

Im Sommer 2017 wurde der im Rahmen der Erhebungsperiode stärkste durchschnittliche P. ulmi-Befall festgestellt: In 20\% der Apfelanlagen wurde ein Drei-Monatsdurchschnitt $>2$ Individuen pro Blatt verzeichnet. Der Median des Durchschnittsbefalls lag bei 0,09 Individuen pro Blatt ( $\mathrm{Max}=6,37$ ), die Besatzdichte an $P$. ulmi verblieb damit an der Mehrzahl der Apfelanlagen auf einem, bezüglich Schadwirkung, erfahrungsgemäß unerheblichem Niveau [16] [17]. Der kritische Schwellenwert für den $P$. ulmi-Befall unterliegt einer zeitlichen Dynamik: Bis Ende Juni liegt die Toleranzschwelle im Bereich von zwei bis drei Individuen pro Blatt, im Juli bei fünf Individuen pro Blatt und im August sollte die Dichte unter 7,5 Individuen pro

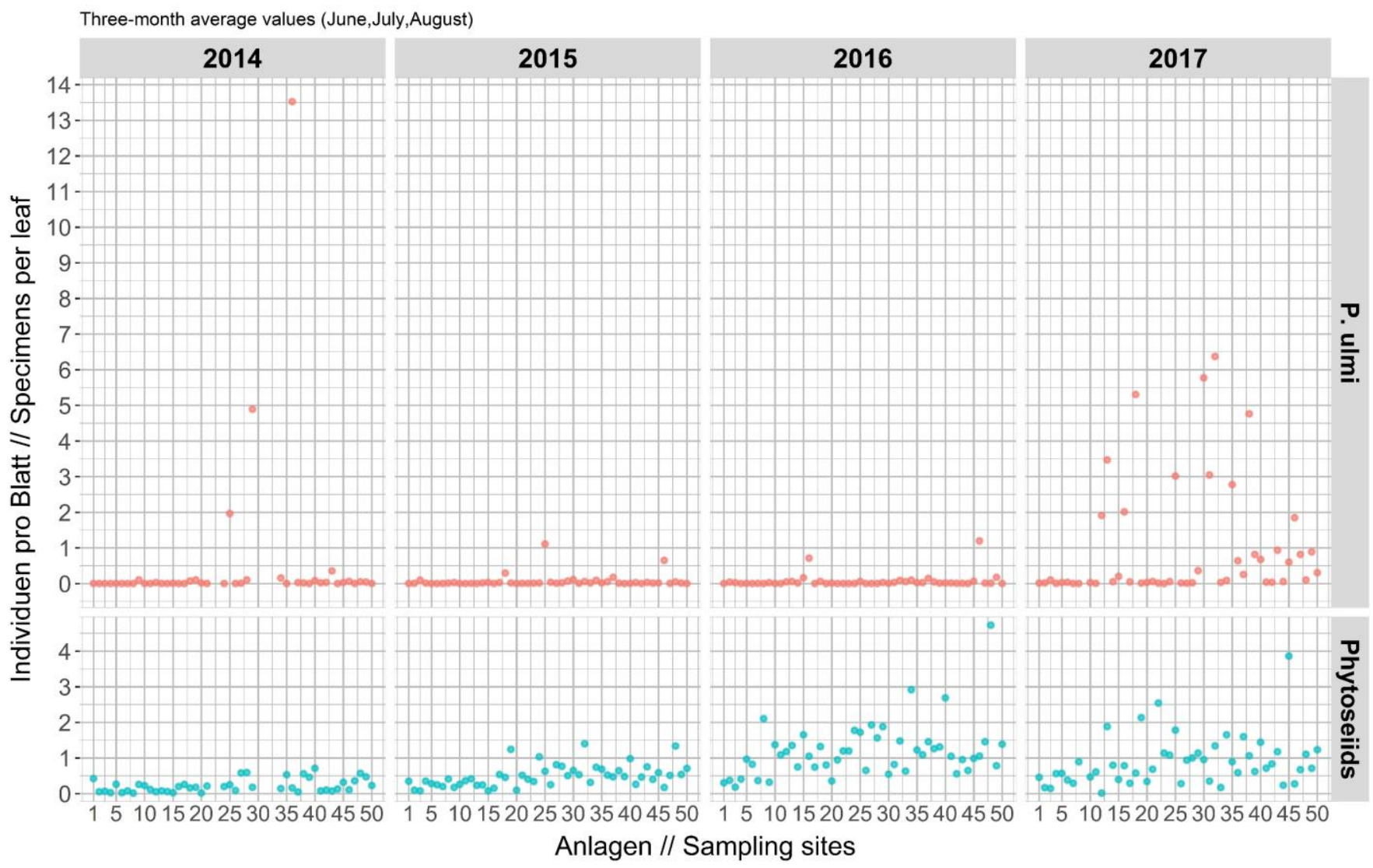

Abb. 3: Populationsdichten von Raubmilben (Phytoseiids) und Panonychus (P.) ulmi der Jahre 2014 -2017. Dichte in Individuen pro Blatt, Datengrundlage: Drei-Monatsdurchschnitt (Juni, Juli \& August). // Population densities of phytoseiid mites and P. ulmi in specimens per leaf for the years 2014-2017 (three-month average values: June, July, August). 
Blatt liegen [18] [19] [20]. Eine erhöhte Anzahl an Individuen wurde überwiegend im Monat Juni und teilweise im Juli festgestellt. Ein im Jahresverlauf kontinuierlicher Befallsdruck konnte nicht festgestellt werden, im Verlauf zum August sank die Populationsdichte generell $a b$.

\section{DISKUSSION}

\section{FAUNISTIK}

Durch die faunistische Erhebung in 27 Apfelanlagen konnten sieben Phytoseiiden-Arten nachgewiesen werden: Amblyseius andersoni (Chant 1957), Neoseiulus californicus (McGregor 1954), Euseius finlandicus (Oudemans 1915), Kampimodromus aberrans (Oudemans 1930), Paraseiulus talbii (AthiasHenriot 1960), Typhlodromus (Anthoseius) bakeri (Garman 1948) und Typhlodromus pyri (Scheuten 1857).

Ein Vorkommen von N. californicus und $T$. bakeri am Apfel ist bereits aus anderen europäischen Anbaugebieten bekannt, wurde hiermit aber erstmals im Südtiroler Raum beobachtet. Die restlichen fünf Arten wurden bereits bei vorangegangenen Untersuchungen festgestellt [13] [14]. Im Gegensatz zu früheren Untersuchungen konnten Paraseiulus soleiger (Ribaga 1902), Paraseiulus triporus (Chant et Shaul 1982) und Phytoseius cf. macropilis (Banks 1909) nicht nachgewiesen werden [5] [6].

Die beobachtete Artenzusammensetzung am Apfel deckt sich mit Berichten aus anderen italienischen Anbaugebieten [21]. Prinzipiell unterschied sich das Artenspektrum an Blättern und Aststummeln nicht wesentlich.

A. andersoni wurde in sämtlichen Apfelanlagen gefunden und erwies sich als eine im Untersuchungsgebiet dominierende Art. Ein alleiniges Auftreten von $A$. andersoni an Aststummeln wurde insbesondere im Raum Meran festgestellt.

T. pyri stellte die zweithäufigste Raubmilbenart dar und konnte an der Mehrzahl der Standorte wenigstens einmalig gefunden werden, in den höheren Lagen des Vinschgaus war sie dominierend. Die Arten K. $a b$ errans und $P$. talbii konnten nur sporadisch nachgewiesen werden, wobei erstere nur an Blättern und letztere überwiegend in Astproben festgestellt wurde.

Die Beobachtung, dass K. aberrans und $N$. californicus ausschließlich an Blättern auftraten, könnte auf ein sporadisches oder zeitlich begrenztes Vorkommen in Südtiroler Apfelanlagen hindeuten. Darüber hinaus könnte das Nicht-Vorkommen an Aststrukturen auf ein alternatives Überwinterungsverhalten dieser Arten hinweisen. So wurde berichtet, dass $N$. californicus diskontinuierlich am Apfel siedelt und zur Überwinterung an die Unterwuchsvegetation migriert [22].

Für Südtirol waren bis dato keine Funde von $N$. californicus am Apfel beschrieben.

Im Untersuchungsgebiet erwies sich das Artenbild als recht uniform, nur im oberen Vinschgau zeigte sich eine gewisse Dominanzverschiebung zugunsten T. pyri. Welche Einflussfaktoren für die beobachtete Artenzusammensetzung und Dominanzstruktur maßgeblich waren, ist nicht bekannt. Diverse biotische sowie abiotische Faktoren können einflussgebend auf Art- und Populationsebene wirken: Klimatische Gegebenheiten, vegetationsbedingte Eigenschaften (Unterwuchszusammensetzung, Blatteigenschaften), Nahrungsangebot (alternative Futterquellen, Pollenangebot), Einsatz von Pflanzenschutzmittel sowie intra- und interspezifische Wechselwirkungen [23] [24] [25] [26] [27].

Bei vielen Vertretern der Phytoseiidae handelt es sich um fakultative Räuber von Kleinarthropoden, deren Diät auch aus pilzlicher und pflanzlicher Kost bestehen kann. Zum Nahrungsspektrum generalistischer Raubmilben zählen unter anderem Pollen, Pflanzenexudate, Pilzsporen (Mehltau-Pilze) sowie diverse Schadmilben und indifferente Milben [28]. Kannibalismus und interspezifische Prädation sind innerhalb der trophischen Gilde der Raubmilden verbreitet [24] [28] [29] [30] [31] [32].

A. andersoni weist ein höheres interspezifisches Prädationsvermögen als die potenzielle Konkurrenzart T. pyri auf [33] [34] [35]. Vrie et Kropczynska, 1967 [36] berichten, dass besonders Larvenstadien und zu einem geringen Teil Adulte von $P$. ulmi zum Beutespektrum von $A$. andersoni zählen, während Eier verschmäht werden. Durch diese bevorzugte Prädation spezifischer Entwicklungsstadien wird ein gänzlicher Zusammenbruch der Beutepopulation vermieden [36] [37]. Die hohe interspezifische Kompetenz sowie die bekannten Futterpräferenzen sind somit Eigenschaften, die möglicherweise zur beobachteten Eudominanz und zum konstanten Vorkommen beitragen.
T. bakeri wurde einmalig und ausschließlich an Aststummeln einer biologisch bewirtschafteten Anlage gefunden. Diese Beobachtung steht in Einklang mit Berichten, wonach $T$. bakeri als Rinden-Bewohner am Apfel und anderen Bäumen festgestellt wurde [9] [38] [39].

Mit Ausnahme von N. californicus und P. talbii handelt es sich bei sämtlichen festgestellten Raubmilbenarten um sogenannte Generalisten, welche diverse Nahrungsquellen nutzen können. $A$. andersoni, $K$. aberrans und $T$. pyri werden gemäß ihrer Futterpräferenzen als generelle Prädatoren eingeordnet, E. finlandicus hingegen ist ein pollenfressender Generalist [29] [40].

P. talbii gilt als spezialisierter Räuber von Milben aus der Familie der Tydeidae, sein Vorkommen ist insbesondere im Europäischen Weinbau dokumentiert [10] [28] [41].

Gemäß McMurtry et Croft, 1997 [29] handelt es sich bei $N$. californicus um einen spezialisierten Räuber, dessen Nahrungsspektrum vor allem Tetranychiden umfasst. In Croft et al., 1998 [42] wird die entsprechende Art hingegen als eine Zwischenform eines spezialisierten und generalistischen Räubers angesehen.

Im Untersuchungsgebiet dominierten ausschließlich Generalisten. Generalistische Räuber vermögen auch in Abwesenheit von großen Beutepopulationen mittels alternativer Futterquellen zu überdauern, während der Bestand von Nahrungsspezialisten unmittelbar an die Beuteressource gebunden ist [30] [43] [44]. Das Überwiegen von Generalisten gründet unter anderem auf ihre breite trophische Variabilität und die damit verbundene Fähigkeit zur interspezifischen Beeinflussung spezialisierter Konkurrenzarten [24] [29] [30] [31] [32].

Schnittstellen, bzw.die daran befindlichen Fissuren, dienen weiblichen Adulttieren als Überwinterungsquartiere. Im Zuge der Untersuchung konnte gezeigt werden, dass die am Blatt dominierenden Arten auch im Winter am Holz der Kultur siedeln. Das dominante Auftreten von A. andersoni und T. pyri an Aststummeln war stets mit einem Vorkommen dieser Arten an Blättern in der darauffolgenden Vegetationsperiode verbunden.

\section{POPULATIONSDICHTEN}

Im Sommer 2014 wurde eine im Mehrjahresvergleich geringe Dichte von $P$. ulmi und 
Raubmilben festgestellt. Eine spezifische Ursache für diese geringen Populationsdichten lässt sich nicht zweifelsfrei feststellen, zumal diese Ausprägung klimatisch bedingten Jahreseffekten, wie auch einer natürlichen Populationsdynamik zugrunde liegen kann.

Im Untersuchungszeitraum 2017 wurde ein im Jahresvergleich erhöhter, jedoch vom Schadpotential erfahrungsgemäß unerheblicher P. ulmi-Befall verzeichnet [16] [17]. Welche Faktoren die beobachtete Populationsentwicklung im Sommer 2017 maßgeblich beeinflussten, kann nicht beurteilt werden, allerdings könnten die klimatischen Gegebenheiten in diesem Jahr einen förderlichen Effekt ausgeübt haben. Die Temperatur im Frühjahr 2017 war vergleichsweise mild. Insbesondere der Mai erwies sich als besonders warm: die Durchschnittstemperatur an der Wetterstation Laimburg lag $1,6^{\circ} \mathrm{C}$ über dem langjährigen Durchschnitt [45].

Die Spinnmilben-Befallssituation im Untersuchungsgebiet (50 Apfelanlagen) unterlag über die Jahre Schwankungen, erwies sich jedoch stets als unbedenklich. Der kritische Schwellenwert für den $P$. ulmi-Befall unterliegt einer zeitlichen Dynamik: Bis Ende Juni liegt die Toleranzschwelle im Bereich von zwei bis drei Individuen pro Blatt, im Juli bei fünf Individuen pro Blatt und im August sollte die Dichte unter 7,5 Individuen pro Blatt liegen [18] [19] [20].

Raubmilben konnten an sämtlichen Standorten gefunden werden. Im Untersuchungszeitraum 2016 und 2017 wurde in der Mehrzahl der Apfelanlagen eine für die natürliche Spinnmilbenregulation günstige Dichte von $\geq 0,5$ Raubmilben pro Blatt verzeichnet [46]. Das festgestellte Räuber-Beute-Verhältnis war, mit Ausnahme von zwei Apfelanlagen im Jahr 2014, durchwegs > 1:10 (Raubmilben / P. ulmi) und damit in einem gemäß van der Werf et al., 1994 [47] stabilen autoregulatorischen Bereich.

\section{DANK}

Danken möchten wir Luca Gambarotto, Angelika Gruber, Robert Stocker, Manuel Strickner und Johanna Ursch für die Mitarbeit im Freiland und Labor, dem Südtiroler Apfelkonsortium und der Abteilung 31 Landwirtschaft der Autonomen Provinz Bozen Südtirol für die finanzielle Unterstützung.

\section{ZUSAMMENFASSUNG}

Von 2014 bis 2017 wurden intensive Untersuchungen zum Raubmilbenvorkommen (Mesostigmata: Phytoseiidae) und Auftreten der Roten Spinne Panonychus ulmi (Prostigmata: Tetranychidae) in ausgewählten Apfelanalgen in Südtirol (Italien) angestellt. Die Populationsdichten der Milben wurden wiederholt an bis zu 50 Standorten im Etschtal und Vinschgau erhoben. In 27 Apfelanlagen wurde im Verlauf der Jahre 2015-2017 die Artenzusammensetzung blattbesiedelnder sowie an Aststrukturen überwinternder Raubmilben eingehend untersucht. Insgesamt wurden 5581 Raubmilben einer morphologischen Artbestimmung unterzogen. Sechs Phytoseiiden-Arten wurden nachgewiesen. Amblyseius andersoni (Chant 1957) konnte an allen Untersuchungstandorten gefunden werden und wies eine Individuendominanz von $80 \%$ auf. Ein permanentes Vorkommen von Typhlodromus pyri (Scheuten 1857) und Euseius finlandicus (Oudemans 1915) wurde an $18,5 \%$ bzw. $7,4 \%$ der Standorte festgestellt. Kampimodromus aberrans (Oudemans 1930), Paraseiulus talbii (Athias-Henriot 1960) und Typhlodromus bakeri (Garman 1948) wurden sporadisch nachgewiesen. Neoseiulus californicus (McGregor 1954) wurde erstmals am Apfel in Südtirol nachgewiesen. Der P. ulmi-Befall im Untersuchungsgebiet erwies sich, sofern gegeben, als äußerst gering. Eine wesentliche Zunahme der Populationsdichten von $P$. ulmi und eine diesbezügliche Schadwirkung wurden nicht festgestellt.

\section{RIASSUNTO}

È stato condotto un monitoraggio pluriennale (2014-2017) della densità di popolazione degli acari predatori (Mesostigmata: Phytoseiidae) e del ragnetto rosso Panonychus ulmi (Koch 1836) in 50 meleti del Alto Adige. Inoltre è stata svolta un'indagine triennale sulla composizione faunistica degli acari fitoseidi in 27 meleti, situate nel Burgraviato e nella Val Venosta. La composizione delle specie è stata indagata in estate su foglie e durante lo svernamento tramite il campionamento di monconi di ramo. Nel corso delle indagini sono stati identificati 5581 acari fitoseidi appartenenti a sei specie. La specie più comune e abbondante è risultata Amblyseius andersoni (Chant 1957), costantemente presente nel $85 \%$ dei siti indagati. $A$. andersoni è stato reperito in tutti i meleti esaminati e costituiva l' $80 \%$ del numero totale di individui. Una presenza costante di Typhlodromus pyri (Scheuten 1857) e di Euseius finlandicus (Oudemans 1915) è stata accertata rispettivamente nel $18,5 \%$ e 7,4\% dei siti. Le specie Kampimodromus aberrans (Oudemans 1930), Paraseiulus talbii (Athias-Henriot 1960) e Typhlodromus bakeri (Garman 1948) sono state rinvenute soltanto sporadicamente. Nell'estate 2017 è stata riscontrata per la prima volta in Alto Adige la presenza di Neoseiulus californicus (McGregor 1954) su melo. Si può costatare che nei siti oggetto di studio non si è notato un forte incremento delle infestazioni di $P$. ulmi e che l'abbondanza è rimasta generalmente sotto la soglia d'intervento. 


\section{LITERATUR}

[1] Parth M., Fischnaller S., Messner M. et al. (2018). Rote Spinne-Befall und Vorkommen von Raubmilben in ausgewählten Apfelertragsanlagen. Obstbau-Weinbau 55 (4), 1013

[2] Amin M.M., Mizell R.F., Flowers R.W. (2009). Response of the predatory mite Phytoseiulus macropilis (Acari: Phytoseiidae) to pesticides and kairomones of three spider mite species (Acari: Tetranychidae), and non-prey food. Florida Entomologist 92 (4), 554-562, DOI: 10.1653/024.092.0404.

[3] Duso C., Ahmad S., Tirello P. et al. (2014). The impact of insecticides applied in apple orchards on the predatory mite Kampimodromus aberrans (Acari: Phytoseiidae). Experimental and Applied Acarology 62 (3), 391414, DOI: 10.1007/s10493-013-9741-3.

[4] Güven B., Madanlar N. (2010). Laboratory tests on side effects of pesticides used in peach orchards on the predatory mite Typhlodromus athiasae (Porath and Swirski) (Mesostigmata, Phytoseiidae). IOBC/WPRS Bulletin 55, 49-53.

[5] Oberhofer H., Waldner W. (1986). Natural control of spider mites in the orchards of South Tyrol. In: Dickler E., Blommers L. H. M., Minks A. K. (eds.). Proceedings of the 7th Symposium: "Integrated Plant Protection in Orchards", Wageningen, the Netherlands, August 26-29, 1985. IOBC-WPRS Bulletin 9 (4). Organisation internationale de lutte biologique contre les animaux et les plantes nuisibles, Section régionale ouest paléarctique, Zürich, Switzerland, pp. 17-28.

[6] Sölva J., Zöschg M., Hluchy M. et al. (1997). Predatory phytoseiid mites (Acari: Mesostigmata) in vineyards and fruit orchards in Southern Tyrol. Anzeiger für Schädlingskunde, Pflanzenschutz, Umweltschutz 70 (1), 17-19. DOI: 10.1007/BF02009611.

[7] Boller E. (1984). Eine einfache AusschwemmMethode zur schnellen Erfassung von Raubmilben, Thrips und anderen Kleinarthropoden im Weinbau. Schweizer Zeitschrift für Obst- und Weinbau 120 (1), 16-17.

[8] Hernandes F.A., Kreiter S., Tixier M.-S. (2012). The first electronic polytomous key to the world species of the subgenus Typhlodromus (Anthoseius) De Leon (Acari: Phytoseiidae). Zootaxa 3451 (1), DOI: 10.11646/zootaxa.3451.1.4.

[9] Miedema E. (1987). Survey of phytoseiid mites (Acari: Phytoseiidae) in orchards and surrounding vegetation of northwestern Europe, especially in the Netherlands. Keys, descriptions and figures. Netherlands Journal of Plant Pathology 93, 1-63, DOI: 10.1007/BF01984462.
[10] Tixier M.-S., Baldassar A., Duso C. et al. (2013). Phytoseiidae in European grape (Vitis vinifera $\mathrm{L}$.): bio-ecological aspects and keys to species (Acari: Mesostigmata). Zootaxa 3721 (2), DOI: 10.11646/zootaxa.3721.2.1.

[11] Demite P.R., De Moraes G.J., McMurtry, J.A. et al. (2019). Phytoseiidae Database. Retrieved August 03, 2019, from http://www.lea.esalq.usp.br/phytoseiidae/.

[12] Engelmann H. (1978). Zur Dominanzklassifizierung von Bodenarthropoden. Pedobiologica $18,378-380$.

[13] Mühlenberg M., Bogenrieder A. (1993). Freilandökologie. Quelle \& Meyer, Heidelberg/Wiesbaden, Deutschland.

[14] Smith T.M., Smith R.L. $\left(2009^{6}\right)$. Ökologie. Pearson Studium, München, Deutschland, p. 445.

[15] Lehmann G. (1984). Möglichkeiten der Erhebung und Darstellung der Abundanz bei Libellen. Libellula $3(1 / 2), 10-19$.

[16] Höhn H., Annaheim K., Noser S. et al. (2010). Ohne Raubmilben geht's nicht! Schweizerische Zeitschrift für Obst- und Weinbau 146 (19), 8-11.

[17] Wiman N., Stove H. (2020). Tree Fruit Crops: Apple Pests. PNW Insect Management Handbook. A Pacific Northwest Extension Publication, p. J20. Retrieved Jannuary 28, 2021, from https://pnwhandbooks.org/sites/pnwhandbooks/files/insect/chapterpdf/insect20-i-tree-fruits.pdf.

[18] Agnello A., Reissig H., Dunham D. et al. (1999). Early season acaricides to control mites, 1998. Arthropod Management Tests 24 (1), DOI:10.1093/amt/24.1.A1.

[19] Breth D.I., Nyrop J.P., Kovach J. (1998). Achieving Biological Control of European Red Mite in Northeast Apples. An Implementation Guide for Growers. Cornell Cooperative Extension Tree Fruit Crops IPM Publication 215. Retrieved December 5, 2018, from https://hdl.handle.net/1813/43090.

[20] Cross J.V., Berrie A.M. (1994). Sampling and assessment of pests and diseases as the basis for decision making in orchards in the UK. Aspects of applied biology 37, 225-236.

[21] Duso C., Sbrissa F. (1990). Phytoseiid mites (Acari Phytoseiidae) in apple orchards in northern Italy: distribution, biology, ecology and economic importance. Bollettino di Zoologia Agraria e di Bachicoltura 22 (1), 53-89.

[22] Auger P., Tixier M.-S., Kreiter S., Fauvel G. (1999). Factors affecting ambulatory dispersal in the predaceous mite Neoseiulus californicus (Acari: Phytoseiidae). Experimental and Applied Acarology 23 (3), 235-250, DOI: 10.1023/A:1006019014708.
[23] Duso C., Fanti M., Pozzebon A. et al. (2009). Is the predatory mite Kampimodromus aberrans a candidate for the control of phytophagous mites in European apple orchards? $\mathrm{Bi}$ oControl 54, 369-382, DOI: 10.1007/s10526008-9177-6.

[24] Lorenzon M., Pozzebon A., Duso C. (2012). Effects of potential food sources on biological and demographic parameters of the predatory mites Kampimodromus aberrans, Typhlodromus pyri and Amblyseius andersoni. Experimental and Applied Acarology 58 (3), 259-278. DOI: 10.1007/s10493-0129580-7.

[25] Slone D.H., Croft B.A. (2001). Species association among predaceous and phytophagous apple mites (Acari: Eriophyidae, Phytoseiidae, Stigmaeidae, Tetranychidae). Experimental and Applied Acarology 25, 109-126, DOI: 10.1023/A:1010640631355.

[26] Tixier M.-S., Kreiter S., Barbar Z. et al. (2006). Abundance and diversity of phytoseiid mite communities in two arboreta in the south of France. Acarologia 46 (3-4), 169-179, retrieved September 2, 2019, from http://www1.montpellier.inra.fr/CBGP/acarologia/export pdf.php?id=5\&typefile=1.

[27] Tuovinen, T. (1994). Influence of surrounding trees and bushes on the phytoseiid mite fauna on apple orchard trees in Finland. $\mathrm{Ag}$ riculture, Ecosystems and Environment 50 (1), 39-47, DOI: 10.1016/0167 8809(94)90123-6.

[28] McMurtry J.A., De Moraes G.J., Sourassou N.F. (2013). Revision of the lifestyles of phytoseiid mites (Acari: Phytoseiidae) and implications for biological control strategies. Systematic and Applied Acarology 18 (4), 297320, DOI: 10.11158/saa.18.4.1.

[29] McMurtry J.A., Croft B.A. (1997). Life-styles of phytoseiid mites and their roles in biological control. Annual review of entomology 42:291-321, DOI: 10.1146/annurev.ento.42.1.291.

[30] McMurtry J.A. (1992). Dynamics and potential impact of 'generalist'phytoseiids in agroecosystems and possibilities for establishment of exotic species. Experimental and Applied Acarology 14 (3-4), 371-382, DOI: 10.1007/BF01200574.

[31] Prischmann D.A., James D.G., Wright L.C. et al. (2006). Effects of Generalist Phytoseiid Mites and Grapevine Canopy Structure on Spider Mite (Acari: Tetranychidae). Environmental Entomology 35 (1), 56-67, DOI: 10.1603/0046-225X-35.1.56.

[32] Croft B.A., Macrae I.V. (1992). Persistence of Typhlodromus pyri and Metaseiulus occidentalis (Acari: Phytoseiidae) on Apple After Inoculative Release and Competition with Zet- 
zellia mali (Acari: Stigmaeidae). Environmental Entomology 21 (5), 1168-1177, DOI: 10.1093/ee/21.5.1168.

[33] Croft B.A., Kim S.S., Kim D.I. (1996). Intraand interspecific predation on four life stage groups by the adult females of Metaseiulus occidentalis, Typhlodromus pyri, Neoseiulus fallacis and Amblyseius andersoni. Experimental and Applied Acarology 20 (8), 435444, DOI: 10.1007/BF00053307.

[34] Croft B.A., Croft M.B. (1996). Intra- and Interspecific Predation Among Adult Female Phytoseiid Mites (Acari: Phytoseiidae). Effects on Survival and Reproduction. Environmental Entomology 25 (4), 853-858, DOI: $10.1093 / \mathrm{ee} / 25.4 .853$.

[35] Schausberger P., Croft B.A. (2000). Cannibalism and intraguild predation among phytoseiid mites. Are aggressiveness and prey preference related to diet specialization? Experimental and Applied Acarology 24 (9), 709-725. DOI: 10.1023/A:1010747208519.

[36] van de Vrie M., Kropczynska D. (1967). The influence of predatory mites on the population development of Panonychus ulmi (Koch) on apple. Entomophaga 3, 77-84.

[37] McMurtry J.A., van de Vrie M. (1973). Predation by Amblyseius potentillae (Garman) on Panonychus ulmi (Koch) in simple ecosystems (Acarina: Phytoseiidae, Tetranychidae). Hilgardia 42 (2), 17-33, DOI: 10.3733/hilg.v42n02p017.
[38] McMurtry J.A., Huffaker C.B., van de Vrie M. (1970). Ecology of tetranychid mites and their natural enemies. A review: I. Tetranychid enemies: Their biological characters and the impact of spray practices. Hilgardia 40 (11), 331-390, DOI: 10.3733/hilg.v40n11p331.

[39] Huffaker C., van de Vrie M., McMurtry J. (1970). Ecology of tetranychid mites and their natural enemies. A review: II. Tetranychid populations and their possible control by predators: An evaluation. Hilgardia 40 (11), 391-458, DOI: 10.3733/hilg.v40n11p391.

[40] Croft B.A., Blackwood J.S., McMurtry J.A. (2004). Classifying life-style types of phytoseiid mites: diagnostic traits. Experimental and Applied Acarology 33 (4), 247-260, DOI: 10.1023/B:APPA.0000038622.26584.82.

[41] Camporese P., Duso C. (1995). Life history and life table parameters of the predatory mite Typhlodromus talbii. Entomologia Experimentalis et Applicata 77 (2), 149-157. DOI: 10.1111/j.1570-7458.1995.tb01995.x.

[42] Croft B.A., Monetti L.N., Pratt P.D. (1998). Comparative Life Histories and Predation Types. Are Neoseiulus californicus and $N$. fallacis (Acari: Phytoseiidae) Similar Type II Selective Predators of Spider Mites? Environmental Entomology 27 (3), 531-538, DOI: 10.1093/ee/27.3.531.
[43] Nyrop J., English-Loeb G., Roda A. (1998). Chapter 17 - Conservation biological control of spider mites in perennial cropping systems. In: Barbosa P. (ed.). Conservation Biological Control. Academic Press, San Diego, CA, USA, pp. 307-333, DOI: 10.1016/B978012078147-8/50063-3.

[44] Nachman G. (1988). Regional persistence of locally unstable predator/prey populations. Experimental and Applied Acarology 5 (3-4), 293-318. DOI: 10.1007/BF02366099.

[45] Ebner I., Thalheimer M. (2018). Die Witterung im Jahr 2017. Obstbau-Weinbau 55 (1) 26-30.

[46] Polk D. (1994). Insect and mite management. In: Berkett L.P. (ed.). Management Guide for Low-Input Sustainable Apple Production. DIANE Publishing, Darby, PA, USA, pp. 51-76.

[47] van der Werf W., Nyrop J.P., Hardman J.M. (1994). Sampling predator/prey ratios to predict cumulative pest density in the mitepredatory mite system Panonychus ulmi Typhlodromus pyri in apples. Aspects of Applied Biology 37, 41-51.

Dieses Werk ist lizenziert unter einer Creative Commons Namensnennung-Nicht kommerziell 4.0 International Lizenz.

Quest'opera è distribuita con Licenza Creative Commons Attribuzione - Non commerciale 4.0 Internazionale.

This work is licensed under a Creative Commons Attribution-NonCommercial 4.0 International License.

Für alle Abbildungen und Tabellen ohne Nennung des Urhebers gilt: ( ) Versuchszentrum Laimburg.

Per tutte le immagini e tabelle senza menzione dell'artefice vale: (C) Centro di Sperimentazione Laimburg.

For all figures and tables without mention of the originator applies: (C) Laimburg Research Centre. 